\title{
Optimal Sensor Communications in Presence of Transmission Delays and Bandwidth Limitations
}

\author{
M. M. Share Pasand and M. Montazeri \\ Department of Electrical and Computer Engineering, Abbaspour Faculty of Engineering, Shahid Beheshti University, Tehran, Iran \\ Correspondence should be addressed to M. M. Share Pasand; m_sharepasand@sbu.ac.ir
}

Received 5 August 2016; Revised 5 November 2016; Accepted 27 November 2016; Published 31 January 2017

Academic Editor: Xiao He

Copyright (c) 2017 M. M. Share Pasand and M. Montazeri. This is an open access article distributed under the Creative Commons Attribution License, which permits unrestricted use, distribution, and reproduction in any medium, provided the original work is properly cited.

\begin{abstract}
In a distributed sensor (and actuator) network, several sensors may be lumped to a single transmitter or communicate via a remote terminal unit. Due to bandwidth limitations in the network, the transmitter/remote terminal should decide on the communication sequence according to which it sends sensor data. This decision must be based on an appropriate performance criterion. In this paper, we formulize this problem and propose a convex optimization scheme to optimize the estimation variance via choosing the most appropriate communication sequence. The derived communication sequence is then used for designing a Kalman filter to estimate states of the underlying system. Both cases of Zero-Order Hold and Reset To Zero output policies are investigated and compared in the simulation.
\end{abstract}

\section{Introduction}

Networks are being used in almost any application which involves a distributed system. Using a shared network results in reduced wiring and maintenance costs [1]. It also facilitates any extension or restructuring of the network [2]. A restrictive limitation of a shared network is the bandwidth limitation which is usually characterized by the maximum number of simultaneous communications permitted by the network medium access policy [3]. The sequence, according to which sensors are granted access to the network, is henceforth called the sensor communication sequence [3, 4]. Choice of the sensor communication sequence affects estimation and control performance. Figure 1 depicts the situation in which several sensors are sending measurements through a shared limited bandwidth network which cause different measurements to reach the destination with different time delays. A similar problem arises in sensor-actuator networks known also as networked control systems where actuator accesses to the network should be arbitrated in order to stabilize a plant or optimize the control performance [2-4]. The problem of choosing the best communication sequence is hybrid involving both continuous and binaryvalued variables [5] and of combinatorial nature [6] in which the number of feasible sequences is of a combinatorial order. In this paper, we use results of $[7,8]$ to formulate the problem as a convex optimization which could be solved via numerically efficient tools like CVX [8] and similar software. In order to formulate the problem into a convex optimization one, binary-valued variables are relaxed and expressed as continuous variables with inequality constraints which results in suboptimal solutions to the original problem.

The scheduler shown in Figure 1 is a functional block representing a medium access policy for arbitration of sensor accesses to the network, for example, via token passing. It is worth noticing that we assume contention-free medium access policies (e.g., field bus networks) in which access to the network could be determined via an algorithm like a round robin or an online programming. This contrasts to contention-based networks where the access to network occurs randomly based on a multiple access policy in which a node can access the network whenever it is idle [6].

The sensor selection/scheduling problem has been studied for a while [7-13]. Different approaches have been 


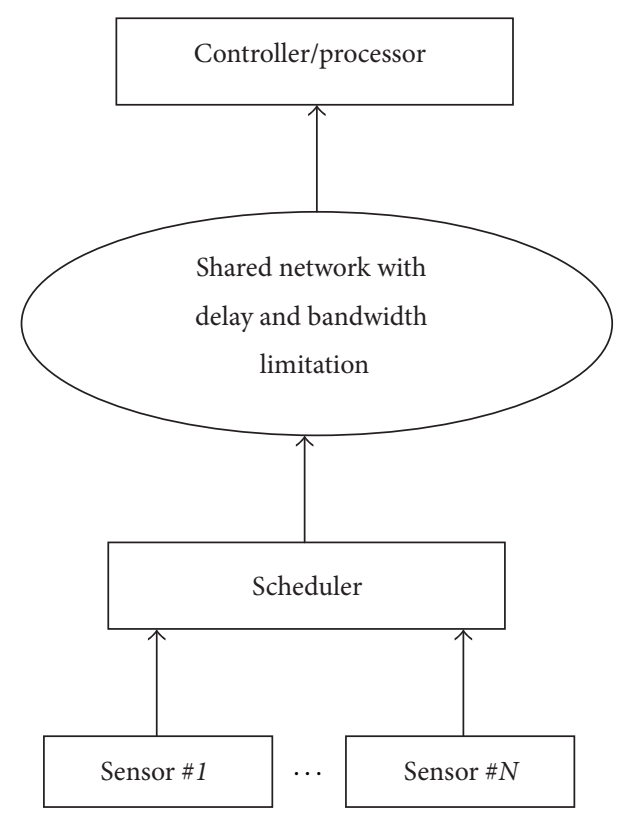

FIgURE 1: Several sensors using a shared network.

practiced for different problem settings in sensor scheduling paradigm. This problem is known to be challenging [7] firstly due to its mixed nature, involving both integer and continuously valued variables $[3,10]$, secondly, as it usually results in a combinatorial optimization problem with severely increasing computational complexity due to the large number of possible schedules in large systems [7], and thirdly due to the complicated relationship between usually used any performance criteria (i.e., logarithmic, quadratic, etc.) and the schedule. This complication hinders the application of conventional optimization methods [7]. It is shown in [12] that most of the conventionally used functions for optimization of sensor schedules are not submodular and, therefore, incremental algorithms could not be used for derivation of optimal schedules in this problem. Though sensor selection problem and sensor scheduling are closely related, some differences should be pointed out. The sensor selection is usually performed offline to select a few sensors out of all available ones [10] in order to achieve optimal estimation. On the other hand, the sensor scheduling is performed offline, online, or semionline in order to determine the order of sensor accesses to network which may be randomly chosen based on a preassigned distribution function [13] or a deterministically (offline or online) assigned schedule as in $[4,5,7]$ and the present paper. Also, the result of a sensor selection algorithm is a subset of all sensors which means some of the sensors are to be discarded and there is no priority between the selected sensors. However, in the sensor scheduling problem, no sensor is discarded and each sensor is granted a portion of the available bandwidth. Some authors [7] however used static sensor scheduling and sensor selection as alternative terms.
In $[7,8,10]$, a static schedule is derived via minimization of the trace of estimation covariance matrix. The schedule is derived offline and does not change during run time. It is known that the estimation error extremely depends on the sensor scheduling [6]. Therefore, the static schedule proposed in [7] lacks the flexibility required for real applications. In fact, we expect a scheduler to reschedule the sensor accesses (either in a time triggered or in event triggered manner) to adequately reject disturbances. Therefore, those methods, suitable for online or semionline implementation like that of [10], are generally preferred in practice. In [9] a periodic schedule is derived via offline optimization of a joint system consisting of scheduling and estimation problems. The bandwidth limited communication is simulated by penalizing the number of communicating sensors in the cost function. Therefore, the number of sensors simultaneously using the network may be different in each time tick. This approach is not suitable in real time networks with bandwidth limitations where the number of sensors capable of communicating simultaneously is bounded by a fixed number and could not be exceeded. In these networks, it is better to model the bandwidth limitation as a hard constraint in the optimization problem or via the use of scheduling matrices (as shown in the next section of this paper).

References $[4,9]$ assume that those channels that have not been updated in the last time tick are Reset to Zero (henceforth referred to as RTZ policy) instead of being held (henceforth referred to as Zero-Order Hold; $\mathrm{ZOH}$ policy). The question of either holding or forgetting sensor or actuator data is discussed in $[4,5]$. While the problem has received attention in the case of actuator scheduling (see $[2,4,14])$, the case of sensor scheduling, apparently, has received less attention. In this paper we assume both policies and compared them to each other in the simulation example. While ZOH seems to be more practical, using RTZ is computationally easier.

It is important to note that this work differs from that of [15-21] and references therein, where it is assumed that all sensor information could be sent to the estimator when network bandwidth is available. This situation is usually referred to as "intermittent" measurement/observation, though the term "scheduled measurement" has been used for this situation in [15]. We also assume that the network is always available to the smart sensor/transmitter but the available bandwidth is limited and constant. Therefore, the smart sensor must decide which of the sensors should be connected to the network at each time tick. The optimal observer gain is derived via periodic Riccati equation which is introduced in [22] and later used in [20, 21]. The main difference between this paper and $[20,21]$ is that the communication sequence is also optimized in this paper. Therefore a twofold optimization is given. Firstly, the sensor communication sequence is optimized. Secondly, the optimal observer is designed for the derived communication sequence. Structural properties of the underlying system are investigated in $[2,6,23]$.

Contributions of the Present Paper. This paper utilizes a convex optimization approach for the derivation of optimal sensor schedule and uses the conventional Kalman filter for 
periodic time-varying systems practiced in $[4,22]$ for optimal state estimation. For this purpose, the optimal schedule is first chosen and incorporated into the plant dynamics. The incorporation of the sensor schedule into the plant model results in a periodic time-varying linear system which is then used as the model for optimal state estimation. Therefore, the state vector of the underlying time invariant discrete time plant is estimated via optimally scheduled measurements. The adopted approach, which facilitates modeling of constant delays (as shown in the sequel) as well as bandwidth limitations, allows for use of existing software in convex programming and simplifies the design procedure. While previous work has considered bandwidth limitations or delays separately, this paper proposes a framework for modeling both constraints simultaneously.

\section{Modeling and Preliminaries}

Consider a Linear Time Invariant (LTI) system subject to bandwidth limited measurements with holding policy in output, described by the following relations:

$$
\begin{aligned}
x(k+1) & =A_{n \times n} x(k)+B u(k), \\
y(k) & =C x(k) \\
\bar{y}(k) & =S_{S}(k) y(k), \\
\check{y}(k) & =\bar{y}(k)+\bar{S}_{S}(k) \check{y}(k-1) .
\end{aligned}
$$

Matrices $A_{n \times n}, B_{n \times n_{u}}$, and $C_{n_{y} \times n}$ are real valued, constant matrices representing state, input, and output matrices of the state space model, respectively. $\bar{y}(k)$ is the transmitted value of output where Reset To Zero (RTZ) policy is in place. $\bar{y}(k)$ contains those elements from $y(k)$ to which medium access is granted with other elements replaced by their most recent (previous) values. $\check{y}(k)$ is the transmitted output when $\mathrm{ZOH}$ policy is used. To establish a relationship between $\bar{y}(k)$ and $y(k)$ the notions of scheduling matrix $S_{S}(k)$ and communication sequence $\sigma_{p s}$ are used.

$$
\begin{aligned}
& S_{S}(i, j)(k) \\
& = \begin{cases}1 & \text { if } i=j \text { and sensor } i \text { is granted access at time } k \\
0 & \text { else. }\end{cases}
\end{aligned}
$$

Due to bandwidth limitation, only $b_{S}$ nodes can access the medium simultaneously; that is

$$
\operatorname{rank}\left(S_{S}(k)\right) \leq b_{S}
$$

A $p$-periodic sensor (output) communication sequence $\sigma_{p s}=$ $\left\{S_{S}(0), \ldots, S_{S}(p-1)\right\}$ represents the order of accesses granted to sensors. This is a binary-valued function denoting the medium access status of the $i$ th output at time ticks $0 \cdots p-1$ and is repeated afterwards. A " 0 " in the $i$ th diagonal element of $S_{S}(k)$ means "accessing the network" and a " 1 " means "not accessing the network."
Remark 1. System description (1)-(2) could be used for modeling constant delays as well. To show this, consider an LTI system with delayed output measurements as follows:

$$
\begin{aligned}
\tilde{x}(k+1) & =\widetilde{A} \tilde{x}(k)+\widetilde{B} \tilde{u}(k), \\
\tilde{y}(k) & =\sum_{i=1, \ldots, N_{d}} \widetilde{C}_{i} \tilde{x}\left(k-d_{i}\right), \quad 0 \leq d_{1} \leq \cdots \leq d_{N_{d}} .
\end{aligned}
$$

Define

$$
\begin{aligned}
x(k) & =\left[\begin{array}{c}
\tilde{x}(k) \\
\tilde{x}(k-1) \\
\vdots \\
\tilde{x}\left(k-d_{N_{d}}\right)
\end{array}\right], \\
A & =\operatorname{diag}(\widetilde{A}), \\
B & =\left[\begin{array}{c}
\widetilde{B} \\
\vdots \\
\widetilde{B}
\end{array}\right], \\
C & =\left[\begin{array}{lll}
C_{1} & \cdots & C_{N_{d}}
\end{array}\right],
\end{aligned}
$$

where $C_{1}=\widetilde{C}_{i}$ if $\widetilde{x}\left(k-d_{i}\right)$ appears in the output and is a zero matrix otherwise. The augmented state space representation is in the form of (1)-(2) and, therefore, is suitable for the methods proposed by this paper.

The following definition is essential to sensor scheduling problem.

Definition 2. A p-periodic communication sequence is called admissible if all sensors are read at least once, during each period.

The admissibility feature of a sensor communication sequence guarantees that the plant preserves its structural properties (i.e., observability, reconstructability, and detectability) after inclusion of the communication sequence into the plant model. (See $[2,4,6]$.)

At each time tick $k$, the observer is to estimate the system state vector based on data received from $b_{S}$ sensors which were granted medium access at the previous time tick. For other sensors, the observer may use data from the last time tick they have been granted access, that is, the most recent data $(\mathrm{ZOH}$ policy), or it may simply forget the last data and uses the newly received output as the current output. Note that the observer will, however, use past data in the observation process, via dynamic equations governing the Kalman filter (see equations (22)-(27)). Therefore, using RTZ does not mean that past data are completely neglected by the observer. Equations (1)-(4) describe the distributed system from the observer point of view. This model incorporated dynamics of the plant together with the access status of the communication medium. The incorporated plant is a timevarying linear system whose parameters are functions of 
the communication sequence. In this setting, it is required to choose a communication sequence (which will control traffic on the shared medium) and the observer (gain) to reconstruct state vector via available measurements. As the communication sequence determines the time-varying dynamics of the resulting incorporated plant, the optimal estimator (including observer dynamics and the chosen communication sequence) depends on the communication sequence. Therefore, a joint problem, that is, optimizing with respect to both estimation and communication, should be solved. A crucial requirement for a communication sequence is that after it is incorporated into the plant dynamics, the incorporated NCS remains observable/detectable. This is addressed in the following Lemma.

Lemma 3. If the pair $(A, C)$ is observable/detectable and $\sigma_{p s}$ is admissible, then the networked system (1)-(2) with either $\mathrm{ZOH}$ or RTZ policies is observable/detectable. Also, the networked system with constant delays and bandwidth limitation will be reconstructible if the pair $(A, C)$ is observable/detectable and $\sigma_{p s}$ is admissible.

Proof. See [2] for ZOH and [4] for RTZ cases.

To choose an appropriate communication sequence, by evaluating performance function, is a tedious and timeconsuming procedure. Therefore, one has to seek simplified versions of the problem, yielding acceptable suboptimal results. In this regard, an "appropriateness measure" is required to compare two or more communication sequences. The estimation error covariance is assumed here as the measure for optimizing communication sequences. The best communication sequence is the one that results in the smallest confidence ellipsoid which is the minimum volume ellipsoid that contains estimation error with a certain probability (i.e., $\varepsilon_{\alpha}=\left\{z \mid z^{T} \Sigma^{-1} z<\alpha\right\}$, where the probability of the estimation error to be contained in the ellipsoid is described via a $X$-squared cumulative distribution function). In connection with this, consider a set of potential measurements, characterized by relation (7). It is desired to choose a subset of measurements (sensors) that minimizes the volume (or mean radius) of the resulting confidence ellipsoid. The following lemma is recalled for this purpose.

Lemma 4. Given m measurements

$$
\begin{aligned}
z_{i} & =a_{i}^{T} X+w_{i} \\
& i=1, \ldots, m, w_{i} \sim N\left(0, \sigma^{2}\right), a_{i} \in V=\left\{v_{1}, \ldots, v_{q}\right\}
\end{aligned}
$$

the MLE and MAP estimates of $X$ are given by

$$
\begin{aligned}
\widehat{X}_{M L E} & =\left(\sigma^{-2} \sum_{i=1}^{m} a_{i} a_{i}^{T}\right)^{-1} \sum_{i=1}^{m} z_{i} a_{i} \\
\widehat{X}_{M A P} & =\left(\sigma^{-2} \sum_{i=1}^{m} a_{i} a_{i}^{T}+\Sigma_{X}^{-1}\right)^{-1} \sum_{i=1}^{m} z_{i} a_{i} .
\end{aligned}
$$

Also, if $\delta_{i}=\{0,1\}$ represents selection of a specific measurement $z_{i}$ from all possible candidate measurements, the problem of choosing $\bar{m}$ measurements out of $m$ possible, to yield a minimum variance estimation based on MAP, could be formulated as

$$
\begin{array}{ll}
\min & \operatorname{tr}\left(\sigma^{-2} \sum_{i=1}^{m} \delta_{i} a_{i} a_{i}^{T}+\Sigma_{X}^{-1}\right) \\
\text { s.t. } & \delta_{i} \in\{0,1\}, \\
& \sum_{i=1}^{q} \delta_{i}=\bar{m} .
\end{array}
$$

By relaxing the nonconvex constraints $\delta_{i} \in\{0,1\}$ to the convex constraints $\delta_{i} \in\left[\begin{array}{ll}0 & 1\end{array}\right]$, one obtains a convex relaxation of (10). The resulting optimization problem, unlike the original sensor selection problem, is a convex optimization problem since the objective function is convex in $\delta_{i}$ and the equality and inequality constraints are linear (in $\delta_{i}$ ). This problem can be solved efficiently, for instance, using interior point methods. (See [10] and references therein.) These methods are known to typically require a few tens of iterations each of which having a complexity of $O\left(\mathrm{~m}^{3}\right)$ [10]. The relaxed problem is not equivalent to the original sensor selection problem; however the optimal objective value of the relaxed problem is a lower bound for the original sensor selection problem as its feasible set contains the feasible set of the original problems.

\section{Sensor Scheduling Problem}

3.1. RTZ Case. The problem of optimal scheduling of sensors in an LTI system described by (1) and (2) could be stated as a convex optimization problem of the following form:

$$
\min \operatorname{tr} \underbrace{\left(\sigma^{-2}\left(\sum_{i=1}^{m} A^{(-m+i-1) T} C^{T}\left(\sum_{j=1}^{q} \delta_{i j} S_{S j}\right) C A^{(-m+i-1)}\right)+\Sigma_{m+1}^{-1}\right)^{-1}}_{\Sigma_{\delta}^{-1}}
$$

$$
\begin{array}{ll}
\text { s.t. } & 0 \leq \delta_{i j}(k) \leq 1, \\
& \sum_{j=1}^{q} \delta_{i j}=1,
\end{array}
$$




$$
\begin{aligned}
& \sum_{i=1}^{m} \sum_{j=1}^{q} \delta_{i j}=\bar{m}, \\
& \sum_{i=1}^{m} \delta_{i j} \geq 1,
\end{aligned}
$$

where

$$
\begin{aligned}
\Sigma_{m+1} & =A^{m} \Sigma_{1}\left(A^{T}\right)^{m}, \\
x(1) & \sim N\left(0, \Sigma_{1}\right), \\
S_{S}(i) & =\sum_{j=1}^{q} \delta_{i j} S_{S j} \quad i=1, \ldots, m .
\end{aligned}
$$

Possible measurements and the set of values for $a_{i}$ are

$$
\begin{aligned}
z_{i} & =\tilde{y}(i) \quad i=1, \ldots, m \\
a_{i} & \in V=\left\{v_{1}, \ldots, v_{m q}\right\} \\
& =\left\{S_{S i} C A^{-j} \mid S_{S i} \in \Psi, 1<i<q, 1<j<m\right\} .
\end{aligned}
$$

$\Psi$ is the set of all possible sensor scheduling matrices. For a bandwidth of $b_{s}$, the number of possible matrices is $\left(\begin{array}{c}n_{y} \\ b_{s}\end{array}\right)$. If some logical constraints on the selected set of sensors exist, for instance, when $q \leq b_{s}$ number of sensors are to be read simultaneously, the number of possible scheduling matrices decreases.

Remark 5. Equations (11)-(12) are a convex optimization problem since the objective function is convex and all constraints could be stated as linear equalities/inequalities.

Adding a slack variable, (12) could be transformed into a semidefinite convex program, as [10]

$$
\begin{array}{ll}
\min & \operatorname{tr} Y \\
\text { s.t. } & 0 \leq \delta_{i}(k) \leq 1 \quad 1 \leq i \leq m, \quad 1 \leq j \leq q \\
& \sum_{j=1}^{q} \delta_{i j}=1 \\
& \sum_{i=1}^{m} \sum_{j=1}^{q} \delta_{i j}=\bar{m} \\
& {\left[\begin{array}{cc}
Y & I \\
I & \Sigma_{\delta}^{-1}
\end{array}\right] \geq 0} \\
& \sum_{i=1}^{m} \delta_{i j} \geq 1 .
\end{array}
$$

It may be of interest to dedicate a specific portion of available accesses to each sensor. Therefore, the last constraint of (15) may be replaced by

$$
\sum_{i=1}^{m} \delta_{i j}>m_{j}
$$

$m_{j}$ represents a portion of $m$ dedicated to the $j$ th sensor.

Remark 6. The proposed formulation could be modified to cover a time-varying state matrix as follows:

$$
\begin{aligned}
& x(k+1)=A_{k} x(k) \\
& \tilde{y}(k)=S_{S}(k) C x(k) \\
& z_{i}=\tilde{y}(i) \quad i=1, \ldots, m \\
& a_{i} \in V=\left\{v_{1}, \ldots, v_{m q}\right\}=\left\{S_{S i} C \prod_{r=1}^{j} A_{m-r+1}^{-1} \mid S_{S i} \in \Psi, 1 \leq i \leq q, 1 \leq j \leq m\right\} \\
& \min \operatorname{tr} \underbrace{\left(\sigma^{-2}\left(\sum_{i=1}^{m} \prod_{r=1}^{m-i+1} A_{r}^{-T} C^{T}\left(\sum_{j=1}^{q} \delta_{i j} S_{S j}\right) C \prod_{r=1}^{m-i+1} A_{r}^{-1}\right)+\Sigma_{m+1}^{-1}\right)^{-1}}_{\Sigma_{\delta}^{-1}} \\
& \text { s.t. } \quad 0 \leq \delta_{i j}(k) \leq 1 \text {, } \\
& \sum_{j=1}^{q} \delta_{i j}=1
\end{aligned}
$$




$$
\begin{gathered}
\sum_{i=1}^{m} \sum_{j=1}^{q} \delta_{i j}=\bar{m}, \\
\sum_{i=1}^{m} \delta_{i j} \geq 1 \\
\Sigma_{m+1}=\prod_{r=1}^{m} A_{r} \Sigma_{1} \prod_{r=1}^{m} A_{r}^{T}, \\
x(1) \sim N^{N}\left(0, \Sigma_{1}\right), \\
S_{S}(i)=\sum_{j=1}^{q} \delta_{i j} S_{S j} ; \quad i=1, \ldots, m .
\end{gathered}
$$

3.1.1. Design Procedure. Using the idea provided in the previous sections, an appropriate schedule could be derived via the following procedure.

Step 1. Solve the optimization problem to find a sequence.

Step 2. Derive a time-varying (periodic) system by including the sequence computed in Step 1, into the plant dynamics.

Step 3. Design a Kalman filter for the system derived is Step 2.

Step 4. Apply the filter for sufficiently large number of time ticks (a multiplication of $m$ ), until the next optimization round.

The optimal estimator for the time-varying plant that resulted from incorporation of network dynamics into the original plant dynamics is the discrete time Kalman filter, described in two recursive steps. In the following, the analytical expressions for the Kalman filter are reviewed:

$$
\begin{aligned}
& P(0 \mid 0)=P_{0}, \\
& \widehat{x}(0 \mid 0)=0 \\
& x(k+1)=A_{k} x(k)+B_{k} u(k) \\
& \tilde{y}(k)=S_{S}(k) C x(k) \\
& P(k+1 \mid k)=A P(k \mid k) A^{T}+Q(k) \\
& P(k+1 \mid k+1) \\
& \quad=\left(C^{T} S_{S}(k+1) R^{-1}(k+1) S_{S}(k+1) C\right. \\
& \left.\quad+P^{-1}(k+1 \mid k)\right)^{-1} \\
& \widehat{x}(k+1 \mid k+1)=A \widehat{x}(k \mid k)+B_{k} u(k)+L(k+1) \\
& \quad \cdot\left(\tilde{y}(k+1)-S_{S}(k) C A \widehat{x}(k \mid k)\right)
\end{aligned}
$$

$$
\begin{gathered}
L(k+1)=P(k+1 \mid k) C^{T} S_{S}(k+1)(R(k+1) \\
\left.+S_{S}(k+1) C P(k+1 \mid k) C^{T} S_{S}(k+1)\right)^{-1} .
\end{gathered}
$$

$L(k)$ is the Kalman gain and $\widehat{x}(t \mid k)$ and $P(t \mid k)$ are the state estimation and covariance matrix of estimation error at time tick $t$ when the estimation is performed using measurements taken at time tick $k$. As the communication sequence is assumed to be periodic, the aforementioned equations become periodic. Discrete time periodic Ricatti equations are discussed in many works. A comprehensive treatment including conditions for existence and convergence of the solutions could be found in [22].

3.2. $\mathrm{ZOH}$ Case. When $\mathrm{ZOH}$ is used in the observer, the system is described as

$$
\begin{aligned}
& x_{a}(k)=\left[\begin{array}{c}
x(k) \\
\check{y}(k-1)
\end{array}\right], \\
& A_{a}(k)=\left[\begin{array}{cc}
A & 0 \\
S_{S}(k) C & \overline{S_{S}}(k)
\end{array}\right], \\
& C_{a}(k)=\left[\begin{array}{ll}
S_{S}(k) C & \overline{S_{S}}(k)
\end{array}\right] .
\end{aligned}
$$

Note that $A_{a}(k)$ is no longer invertible. Therefore, immediate application of (11) or (20) is not possible. In order to resolve this issue, one may construct an augmented system including a period of the communication sequence. More specifically, define

$$
x_{a p}(k)=\left[\begin{array}{c}
x(k) \\
\vdots \\
x(k-p) \\
\check{y}(k-1) \\
\vdots \\
\check{y}(k-p-1)
\end{array}\right] \text {, }
$$




$$
\begin{aligned}
& \check{y}_{a p}(k)=\left[\begin{array}{c}
\check{y}(k) \\
\vdots \\
\check{y}(k-p-1)
\end{array}\right], \\
& A_{a p}(k)=\left[\begin{array}{cc}
A_{11}(k) & 0 \\
A_{21}(k) & A_{22}(k)
\end{array}\right] \\
& A_{11}(k)=\left[\begin{array}{cccc}
A & & & \\
& I & & \\
& & \ddots & \\
& & & I
\end{array}\right] \\
& A_{21}(k)=\left[\begin{array}{llll}
S_{S}(k) C & & \\
& 0 & & \\
& & \ddots & \\
& & S_{S}(k-p-1) C
\end{array}\right] \\
& A_{22}(k)=\left[\begin{array}{cccc}
\overline{S_{S}}(k) & & \\
I & & \\
& \ddots & \\
& & & I \\
0 & & \ldots & \overline{S_{S}}(k-p-1)
\end{array}\right] \text {, }
\end{aligned}
$$

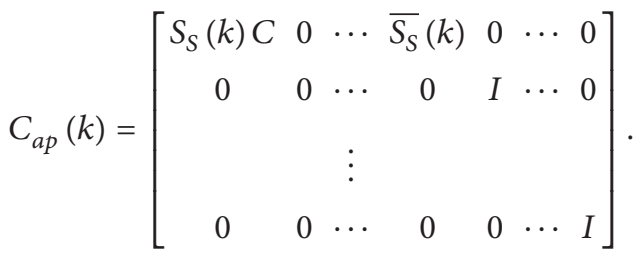

It could be shown that this augmented system is observable if the pair $(A, C)$ is observable and the communication sequence is admissible (see [2]). Also noticing the lower triangular structure of state matrix $A_{a p}(k)$ and counting the number of linearly independent rows provided by identity matrices located in diagonal configuration and the two block rows $\left[\begin{array}{lll}S_{S}(k) C & 0 & \overline{S_{S}}(k)\end{array}\right]$ and $\left[S_{S}(k-p-1) C \quad 0 \quad \overline{S_{S}}(k-p-1)\right]$, it is easily shown that if $A$ is invertible and $C$ is of full-row rank, the matrix $A_{a p}(k)$ will be invertible for all $k$. (If $C$ is not of full-row rank, it will contain linearly dependent rows which implies linearly dependent outputs; by eliminating dependent (redundant) outputs, one may achieve a full-row rank output matrix $C$; as a result, the assumption of having a full-row rank output matrix is not restrictive.)

The resultant augmented system (29) could be used for the scheduling problem (11)-(13) or (15). Kalman filter equations could be applied to (29) after the optimal communication sequence is substituted in the aforementioned equations and system matrices $(A, B)$ replaced by those given in (29). It is straightforward to derive the Kalman gains for (28) from the solutions of the optimal estimation for (29). One may see [3] or [6] for similar treatments.
3.2.1. Further Considerations. Integer $m$ represents the optimization horizon. The lower limit of $m$ is determined by admissibility constraint. $T_{\max }$ is the maximum allowable time between two consecutive readings of a sensor and $T_{\text {cycle }}$ is the minimum time between two consecutive readings on the network. Therefore, one has the following design criterion for choosing $m$ :

$$
\frac{n_{y}}{b_{S}} \leq m \leq \frac{T_{\max }}{T_{\text {cycle }}} .
$$

Resultant $\delta_{i j}$ 's may be close to each other which means no preference exists among several sensors. Therefore, sensors are read based on a round robin schedule until the optimization results in a $\delta_{i j}$ with significant certainty to grant access to the chosen sensor(s).

Note that if the trace of left hand side of (25) is assumed as the cost function as in $[6, c h .6]$, then the optimization problem becomes nonconvex and should be solved using methods like branch and bound [3], genetic algorithms and particle swarm optimization [6], heuristic methods, and tree pruning algorithms (see ch.6 of [6] and references cite therein). However, choosing (19) as the cost function facilitates the use of a convex optimization method, resulting in less computational complexity compared to optimization approaches in previous works including $[6,7,9]$. For MLE estimation, as the current error covariance is not required for optimization, it suffices for the computation time to be smaller than the time required for $m$ communications; that is,

$$
T_{\text {computation }} \leq m T_{\text {cycle }}
$$

\section{Simulation Example}

This section simulates a model for active-passive car suspension system studied in $[3,5,20,21]$. A quarter car model shown in Figure 2 is used for simulation. The quarter car model represents the automotive system at each wheel, that is, the motion of the axle and of the vehicle body at any one of the four wheels of the vehicle. Figure 3 shows the estimation errors for the proposed method: $e_{p}(t)$ and a round robin schedule $e_{r}(t)$ with RTZ policy. Figure 4 shows the estimation error with a round robin; $e_{r z}(t)$ and the proposed method; $e_{p z}(t)$ with $\mathrm{ZOH}$ policy. It is noticed that the proposed method outperforms the round robin. Figure 5 depicts estimation error for the round robin schedule with $\mathrm{ZOH}$ and RTZ policies. Figure 6 shows the state estimation error for the proposed scheduling method with RTZ and $\mathrm{ZOH}$ policies. For both scheduling methods, a Kalman filter is designed based on the time-varying formulation that resulted from incorporating the communication sequence into output equation of (1). Figures 7 and 8 depict the quadratic norm of the estimation errors. According to the estimation error quadratic norms, the proposed method significantly enhanced the estimation. In Figure 9 accesses granted to the first output are shown. It is noticed that in some time ticks the scheduling is different from that of a round robin which has a cyclic pattern with equidistant accesses to 


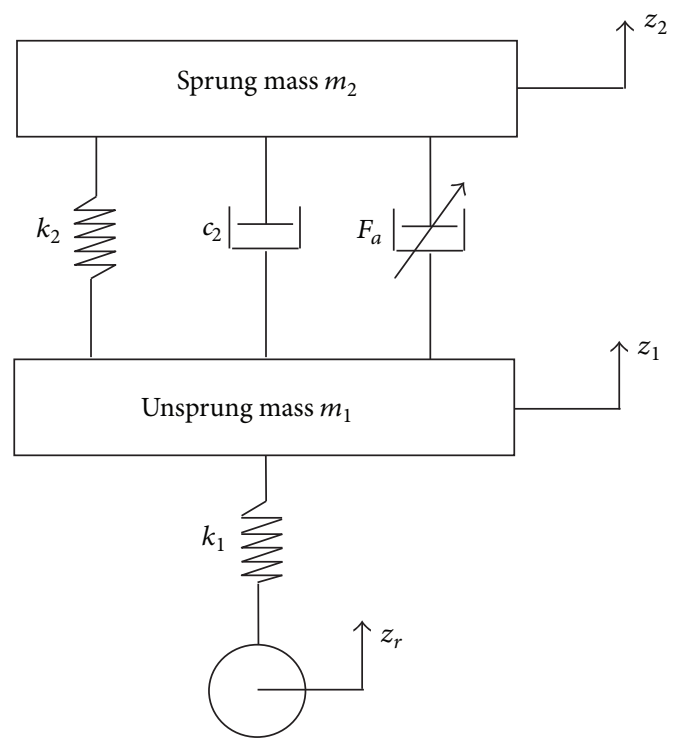

FIGURE 2: The quarter car suspension model.

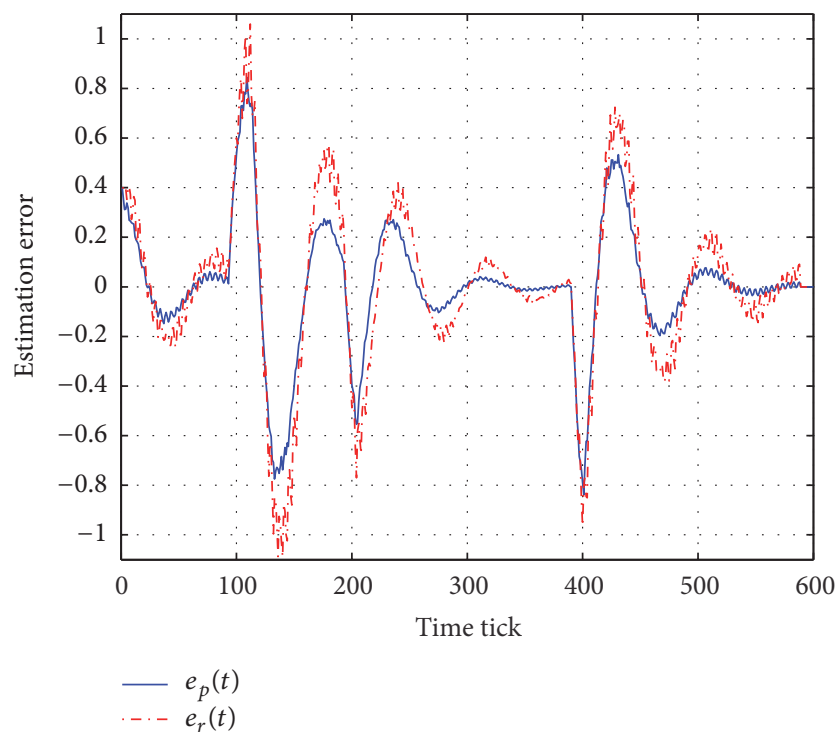

Figure 3: Estimation errors for the proposed method and round robin with RTZ.

each sensor. It is noticed that more accesses are granted to the corresponding output, after scheduling intervals, during $100<k<200$ and $400<k<500$. Simulation parameters are shown in Table 1.

In RTZ policy, the estimation error is deviated more frequently. Also, note that while $\mathrm{ZOH}$ results in a smoother estimation error, the quadratic of the estimation error for RTZ is smaller. This is due to the fact that, in RTZ, outputs are Reset To Zero (and remain zero) until the next access is granted to the corresponding sensor. Quadratic norms of estimation error for the proposed method are significantly smaller than that of RTZ. However, the difference between quadratic norms of estimation errors for $\mathrm{ZOH}$ and RTZ used with the proposed method is not significant (compare the

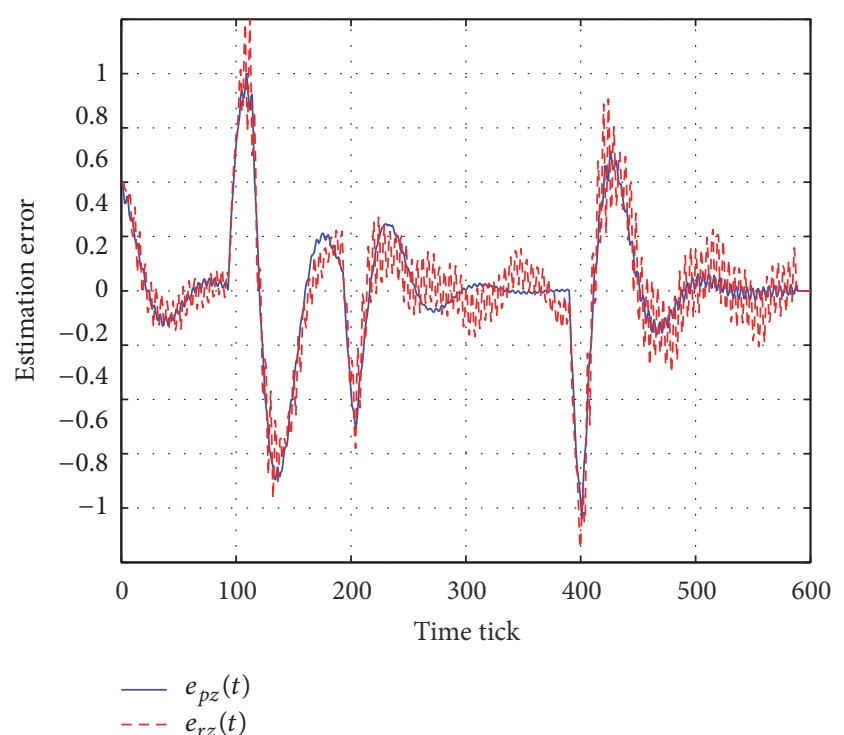

FIGURE 4: Estimation errors for the proposed method and round robin with $\mathrm{ZOH}$.

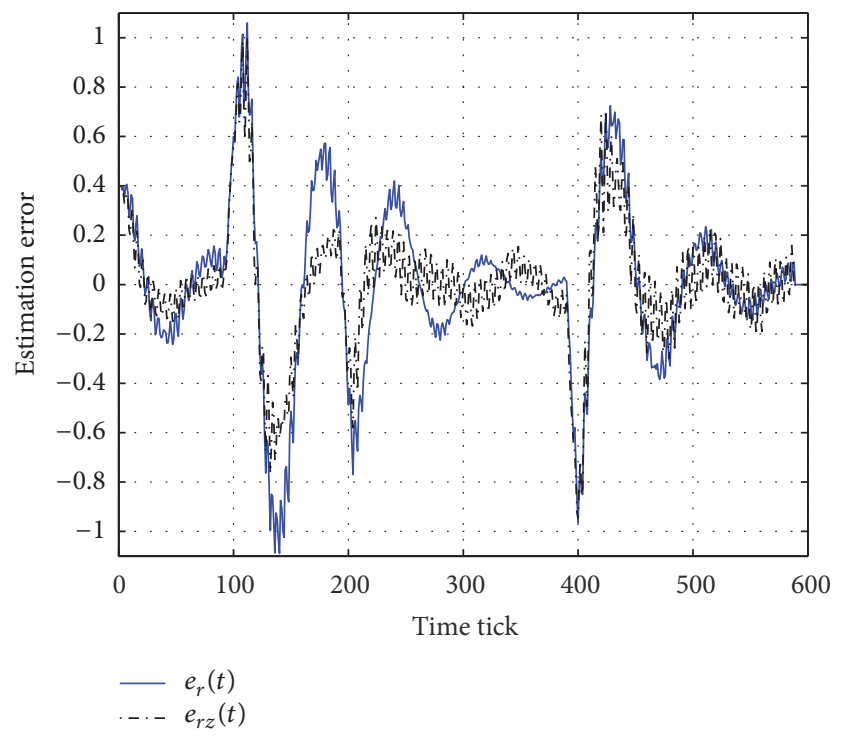

FIGURE 5: Estimation errors for the round robin with RTZ and ZOH.

TABLE 1: Simulation parameters.

\begin{tabular}{lc}
\hline Sample time & $T_{s}=40 \mathrm{~ms}$ \\
Vehicle weight & $M_{s}=1.4$ Tons \\
Unsprung mass (axle and tyre) & $M_{u}=.1$ Tons \\
Spring stiffness & $k_{s}=6 \mathrm{kN} / \mathrm{m}$ \\
Tyre vertical stiffness & $k_{t}=100 \mathrm{kN} / \mathrm{m}$ \\
Process and measurement noise & Negligible \\
Communication sequence period & $m=12$ \\
Scheduling interval & $H=100$ \\
\hline
\end{tabular}

blue curve in Figure 7 with the green curve in Figure 8). In conclusion, the proposed method yields smoother estimation 


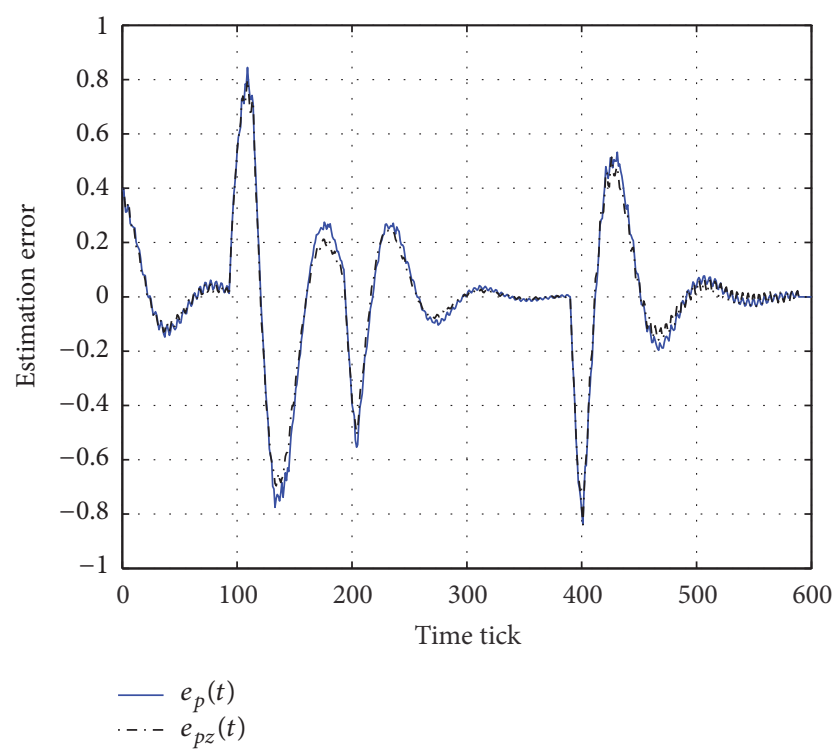

FIGURE 6: Estimation errors for the proposed method with RTZ and $\mathrm{ZOH}$.

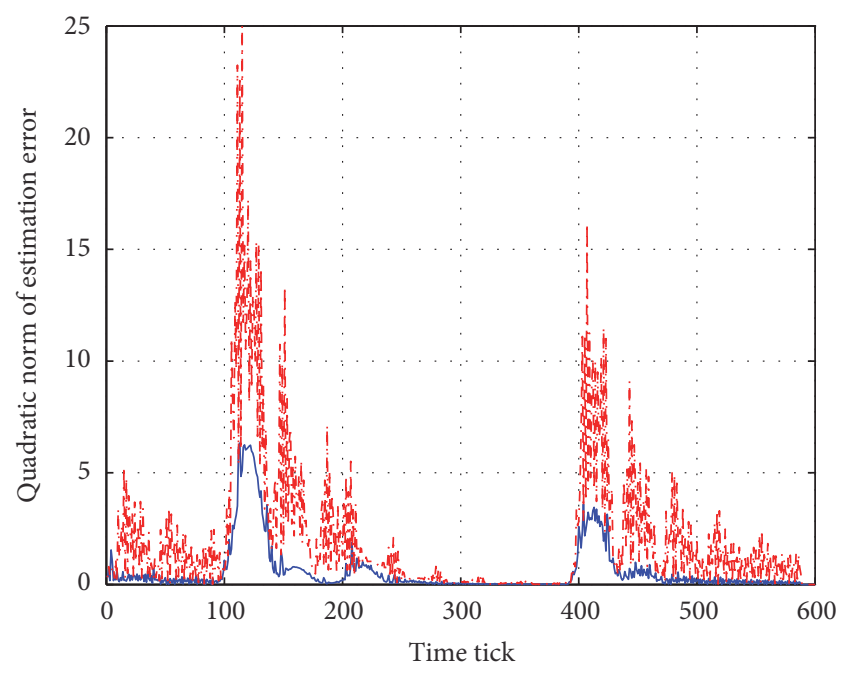

$$
\begin{aligned}
- & e^{2}(t) \\
-\cdot- & e_{r}^{2}(t)
\end{aligned}
$$

FIGURE 7: Quadratic norm of estimation errors for RTZ policy.

with less estimation error. The simulation results are not conclusive about choosing $\mathrm{ZOH}$ or RTZ.

\section{Conclusions}

This paper deals with scheduling of medium access for sensors in a distributed sensor environment. It is assumed that a smart sensor, transmitter, or remote terminal unit is used, capable of arbitrating the network bandwidth among different sensors. A convex optimization approach is proposed for derivation of the optimal schedule. The Kalman filter equations are derived for the resultant periodic system.

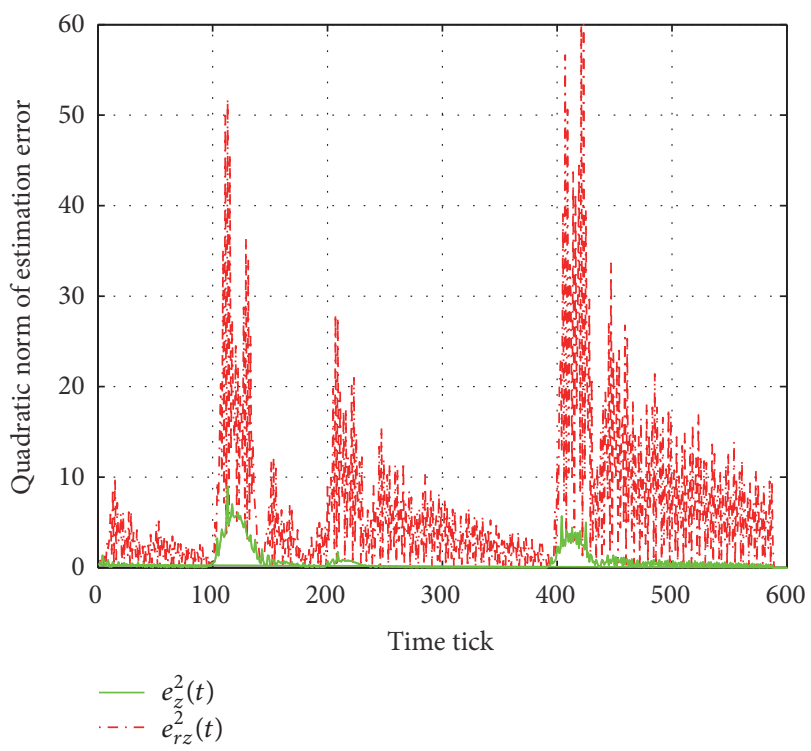

FIGURE 8: Quadratic norm of estimation errors for $\mathrm{ZOH}$ policy.

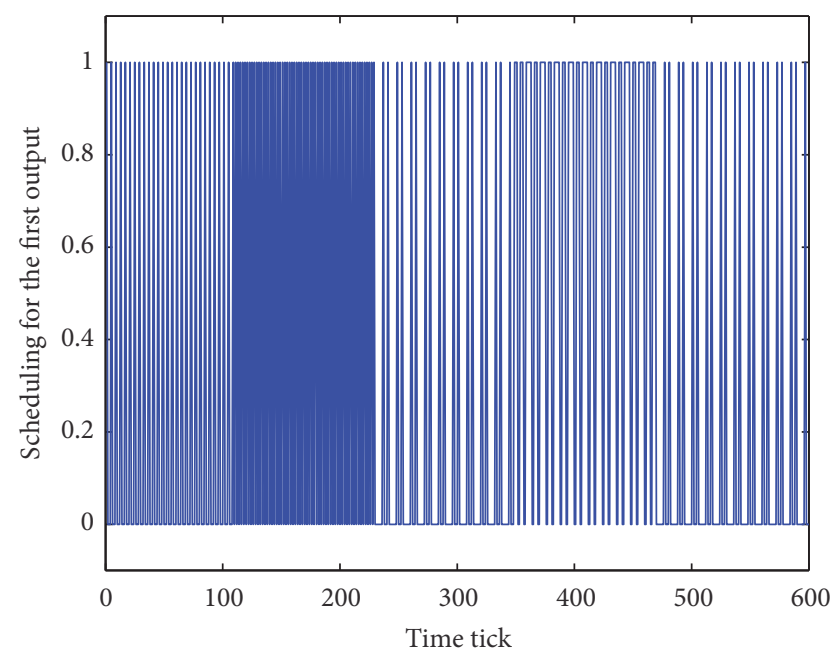

FIGURE 9: Scheduling of $y_{1}(t)$ by the proposed method.

The utilized modeling approach is disjoint and computationally simple compared to previous works which strived to integrate the estimation and scheduling problems into a single optimization framework which could only be solved in offline manner, lacking desirable flexibility. We derived formulations for semionline computation of a periodic output communication sequence specifying the order according to which sensors are to be granted medium access. In order for the optimization problem to be implementable online, instead of summed square estimation error, estimation error covariance at a specific time horizon is considered. The problem of determining optimal sensor communication sequence is formulated as an optimization problem which is then transformed into a convex problem by relaxing the nonconvex constraints into convex constraints. Compared to previously examined approaches, the proposed method has the benefit of convexity and could be solved with less 
computational effort. The proposed method is compared to a round robin schedule with respect to estimation error via a simulation experiment. When the output does not have access to network, it may be kept constant (equal to the last updated value) or Reset To Zero. Both cases are formulated and compared in simulations.

\section{Competing Interests}

The authors declare that they have no competing interests.

\section{References}

[1] J. Baillieul and P. J. Antsaklis, "Control and communication challenges in networked real-time systems," Proceedings of the IEEE, vol. 95, no. 1, pp. 9-28, 2007.

[2] T. Suzuki, M. Kono, N. Takahashi, and O. Sato, "Controllability and stabilizability of a networked control system with periodic communication constraints," Systems and Control Letters, vol. 60, no. 12, pp. 977-984, 2011.

[3] M. E. M. B. Gaid, A. Cela, and Y. Hamam, "Optimal integrated control and scheduling of networked control systems with communication constraints: application to a car suspension system," IEEE Transactions on Control Systems Technology, vol. 14, no. 4, pp. 776-787, 2006.

[4] D. Hristu-Varsakelis and L. Zhang, "LQG control of networked control systems with access constraints and delays," International Journal of Control, vol. 81, no. 8, pp. 1266-1280, 2008.

[5] C. Ionete, A. Cela, and M. B. Gaid, "Controllability and observability of input/output delayed discrete systems," in Proceedings of the IEEE American Control Conference, 2006.

[6] L. Stefano, G. Herrmann, and P. Barber, "Controllability, observability in networked control," IFAC Proceedings, vol. 42, no. 6, pp. 295-300, 2009.

[7] C. Yang, J. Wu, X. Ren, W. Yang, H. Shi, and L. Shi, "Deterministic sensor selection for centralized state estimation under limited communication resource," IEEE Transactions on Signal Processing, vol. 63, no. 9, pp. 2336-2348, 2015.

[8] S. Liu, S. P. Chepuri, M. Fardad, E. Maşazade, G. Leus, and P. K. Varshney, "Sensor selection for estimation with correlated measurement noise," IEEE Transactions on Signal Processing, vol. 64, no. 13, pp. 3509-3522, 2016.

[9] S. Longo, G. Herrmann, and P. Barber, "Robust scheduling of sampled-data networked control systems," IEEE Transactions on Control Systems Technology, vol. 20, no. 6, pp. 1613-1621, 2012.

[10] S. Joshi and S. Boyd, "Sensor selection via convex optimization," IEEE Transactions on Signal Processing, vol. 57, no. 2, pp. 451462, 2009.

[11] T. Sui, K. You, and M. Fu, "Optimal sensor scheduling for state estimation over lossy channel," IET Control Theory \& Applications, vol. 9, no. 16, pp. 2458-2465, 2015.

[12] S. T. Jawaid and S. L. Smith, "Submodularity and greedy algorithms in sensor scheduling for linear dynamical systems," Automatica, vol. 61, pp. 282-288, 2015.

[13] C. Li and N. Elia, "Stochastic sensor scheduling via distributed convex optimization," Automatica, vol. 58, pp. 173-182, 2015.

[14] K. You, N. Xiao, and L. Xie, Analysis and Design of Networked Control Systems, Communications and Control Engineering Series, Springer, London, England, 2015.
[15] S. Kar, B. Sinopoli, and J. M. F. Moura, "Kalman filtering with intermittent observations: weak convergence to a stationary distribution," IEEE Transactions on Automatic Control, vol. 57, no. 2, pp. 405-420, 2012.

[16] Y. Mo and B. Sinopoli, "Kalman filtering with intermittent observations: tail distribution and critical value," IEEE Transactions on Automatic Control, vol. 57, no. 3, pp. 677-689, 2012.

[17] J.-Y. Keller and D. D. Sauter, "Kalman filter for discretetime stochastic linear systems subject to intermittent unknown inputs," Institute of Electrical and Electronics Engineers. Transactions on Automatic Control, vol. 58, no. 7, pp. 1882-1887, 2013.

[18] E. R. Rohr, D. Marelli, and M. Fu, "Kalman filtering with intermittent observations: on the boundedness of the expected error covariance," IEEE Transactions on Automatic Control, vol. 59, no. 10, pp. 2724-2738, 2014.

[19] W. Sun, Y. Zhao, J. Li, L. Zhang, and H. Gao, "Active suspension control with frequency band constraints and actuator input delay," IEEE Transactions on Industrial Electronics, vol. 59, no. 1, pp. 530-537, 2012.

[20] L. Zhang and D. Hristu-Varsakelis, "Communication and control co-design for networked control systems," Automatica, vol. 42, no. 6, pp. 953-958, 2006.

[21] L. Zhang and D. Hristu-Varsakelis, "Stabilization of networked control systems: designing effective communication sequences," in Proceedings of the 16th IFAC World Congress, vol. 38, no. 1, pp. 296-301, 2005.

[22] S. Bittanti, A. J. Laub, and J. C. Willems, Eds., The Riccati Equation, Springer Science \& Business Media, 2012.

[23] D. Hristu-Varsakelis, "Short-period communication and the role of zero-order holding in networked control systems," Institute of Electrical and Electronics Engineers. Transactions on Automatic Control, vol. 53, no. 5, pp. 1285-1290, 2008. 


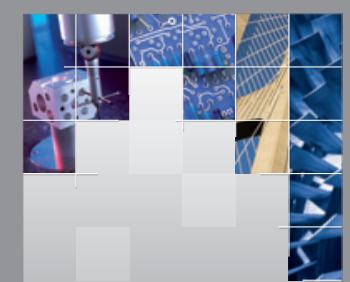

\section{Enfincering}
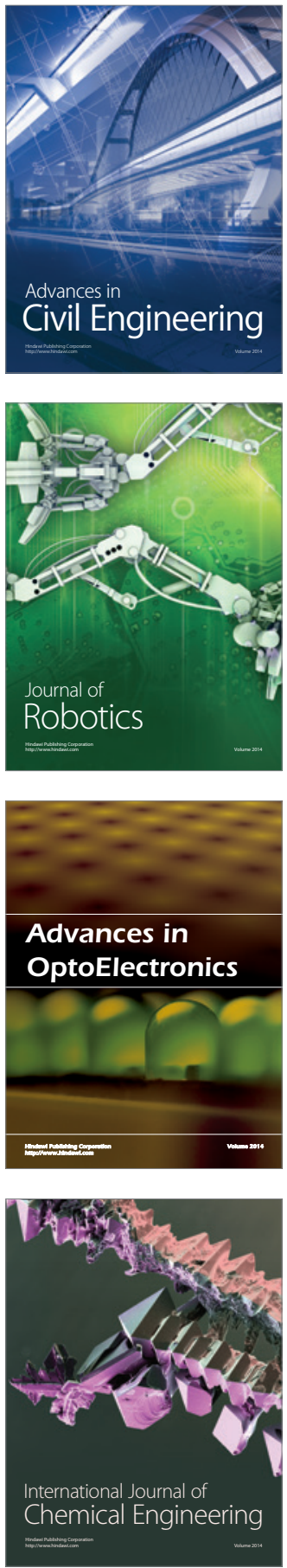

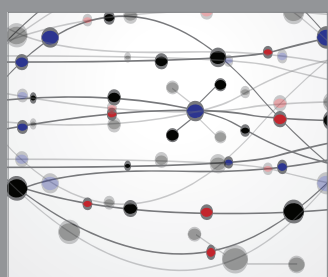

The Scientific World Journal

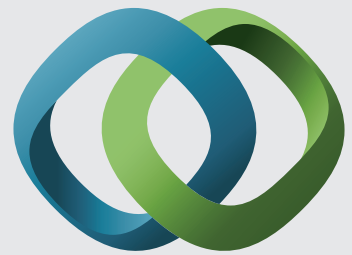

\section{Hindawi}

Submit your manuscripts at

https://www.hindawi.com
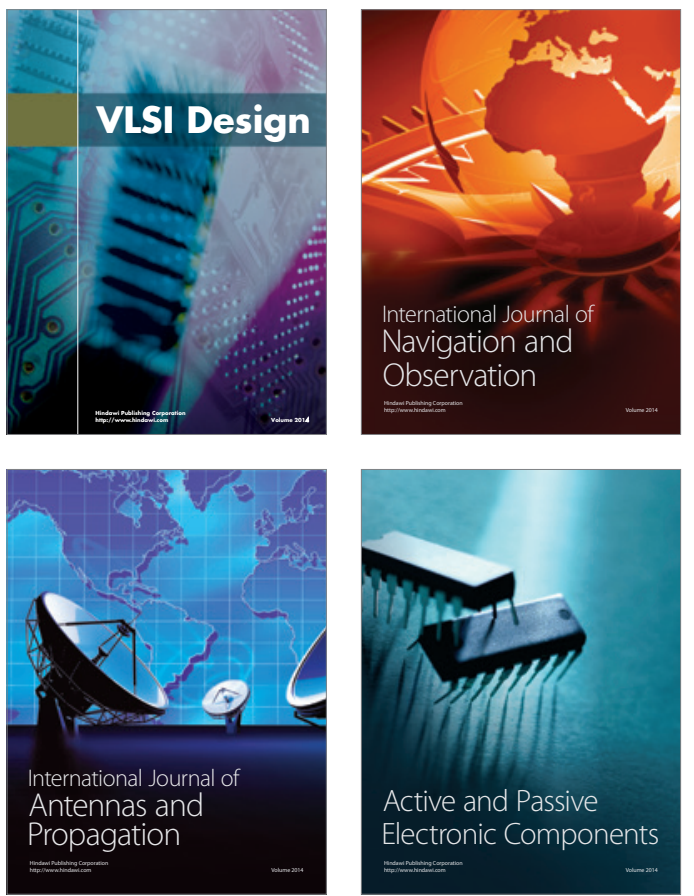
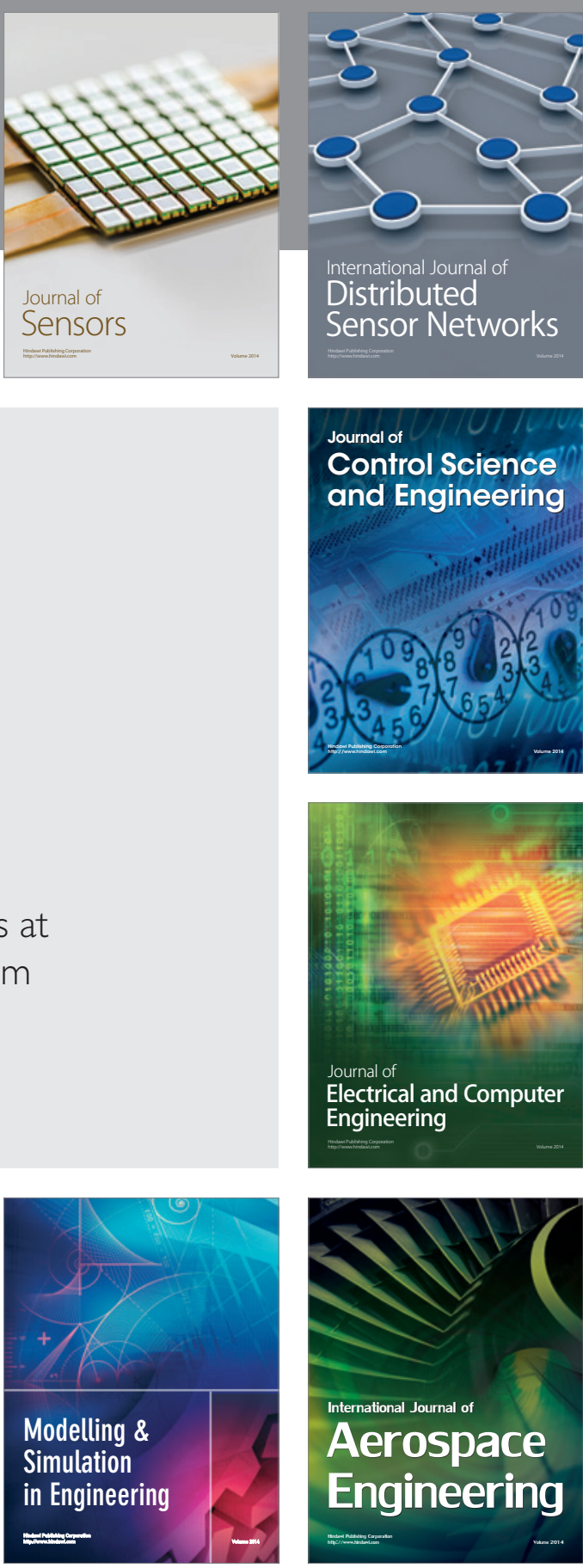

International Journal of

Distributed

Sensor Networks

$-$

Joumal of

Control Science

and Engineering
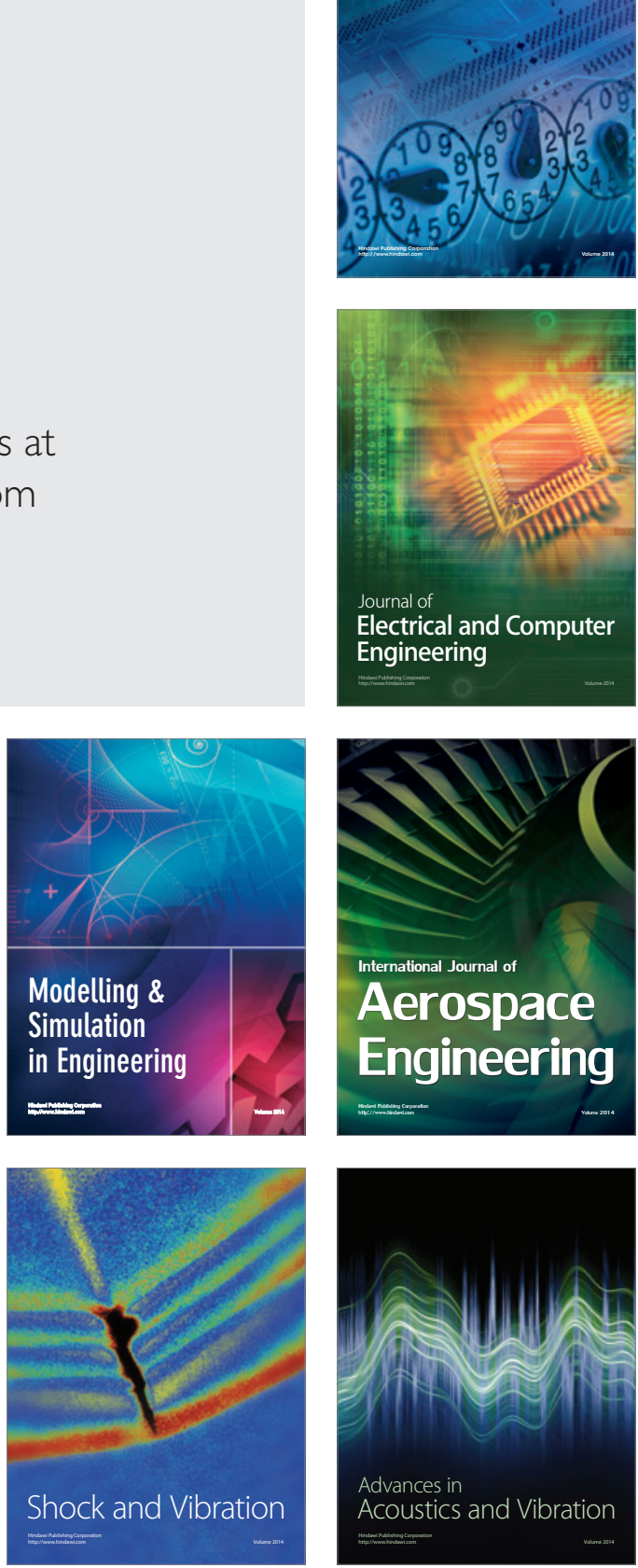